\title{
Regionalisierte Szenarien des Klimawandels
}

\section{Regional climate change scenarios}

Schlagwörter: Klimaänderungen, Klimamodelle, Temperatur, Niederschlag, Regionalisierung

Keywords: Climate change, climate models, temperature, precipitation, regionalisation

\begin{abstract}
Kurzfassung
Der globale Klimawandel bringt auch regionale Veränderungen mit sich, die durch regionale Klimaszenarien berechnet werden. Erste Auswertungen der Modellsimulationen zeigen einen Anstieg der Jahresmitteltemperatur über Deutschland von 2,5 bis $3,5^{\circ} \mathrm{C}$, je nach zugrundeliegendem Emissionsszenario. Regional können maximale winterliche Temperaturanstiege von mehr als $4^{\circ} \mathrm{C}$ im Südosten und Süden Deutschlands erreicht werden. Während sich die Jahresniederschlagssumme in weiten Teilen des Bundesgebietes kaum zu verändern scheint, ist mit einem deutlichen Niederschlagsrückgang während der Sommermonate (bis zu 30\%) und feuchteren Bedingungen während des Winters mit möglichem Ansteigen der Niederschlagssumme um mehr als $30 \% \mathrm{zu}$ rechnen.
\end{abstract}

\begin{abstract}
Global climate change initiates changes in regions as well. These changes can be calculated using regional climate change scenarios. For Germany first results show an increase of the annual mean temperatures between 2.5 and $3.5^{\circ} \mathrm{C}$ depending on the assumed emission scenario. Winter temperatures can rise by about $4^{\circ} \mathrm{C}$ in the South or Southeast of Germany. It seems that the annual precipitation sums do not change, but the annual cycle of precipitation might get more extreme. The results indicate a decrease of summer precipitation up to $30 \%$ in some regions of Germany and an increase in the winter months of more than $30 \%$.
\end{abstract}

\section{Einleitung}

Es ist unumstritten, dass sich das Klima der Erde in den letzten Dekaden gewandelt hat, wie zahlreiche Aufzeichnungen meteorologischer und hydrologischer Dienste weltweit zeigen (Abb. 1). Die globale Mitteltemperatur stieg in der Zeit von 1906 bis 2005 um etwa $0.74^{\circ} \mathrm{C}$, wobei der Trend in der zweiten Hälfte des Zeitraums fast doppelt so groß war wie in der ersten Hälfte (Solomon et al. 2007). Auch in Deutschland hat sich die Lufttemperatur nahe der Erdoberfläche im letzten Jahrhundert um etwa $1^{\circ} \mathrm{C}$ erwärmt und die Jahre 1999, 2000, 2002, 2006, 2007 und 2008 gehören zu den zehn wärmsten Jahren seit 1901 (Daten des DWD 2008).

Von besonderem Interesse ist zusätzlich die Frage, ob, und wenn ja, wie sich extreme bzw. seltene Ereignisse (Starkniederschläge, Hitzewellen, Überschwemmungen, usw.) verändert haben und gegebenenfalls verändern werden. Für derartige Aussagen wurden globale Klimamodelle entwickelt, die zusammen mit verschiedenen Annahmen über die Treibhausgasentwicklung in der Atmosphäre mögliche Entwicklungen des Klimas (Klimaszenarien) in den nächs- ten 100 Jahren berechnen. Diese Computermodelle können als mathematische Abbilder des Erdsystems gesehen werden, da sie die physikalischen Prozesse im Erdsystem numerisch beschreiben und so real wie möglich berechnen. Um die Güte der Klimamodelle einschätzen zu können, werden sie zunächst für die Berechnung vergangener Zeiten eingesetzt. Bevorzugt wird hierzu eine Zeitperiode gewählt, in der zahlreiche Beobachtungen weltweit vorliegen.

\section{Klimaszenarien}

In den letzten Jahrzehnten wuchs in der internationalen Gemeinschaft der Klimaforscher die Sorge, dass menschliche Aktivitäten negative Einflüsse auf das Klima der Erde ausüben könnten. Bereits in einem frühen Stadium der Forschung waren die Wissenschaftler überzeugt, dass der menschliche Einfluss auf das Klima genauer analysiert werden müsse, um die wissenschaftlichen Grundlagen für politische Entscheidungsträger zu schaffen. Dies 
Abbildung 1

Abweichung der globalen Mitteltemperatur $\left(\mathrm{C}^{\circ}\right)$ in $2 \mathrm{~m}$ über Grund von der Referenzperiode 1961 bis 1990

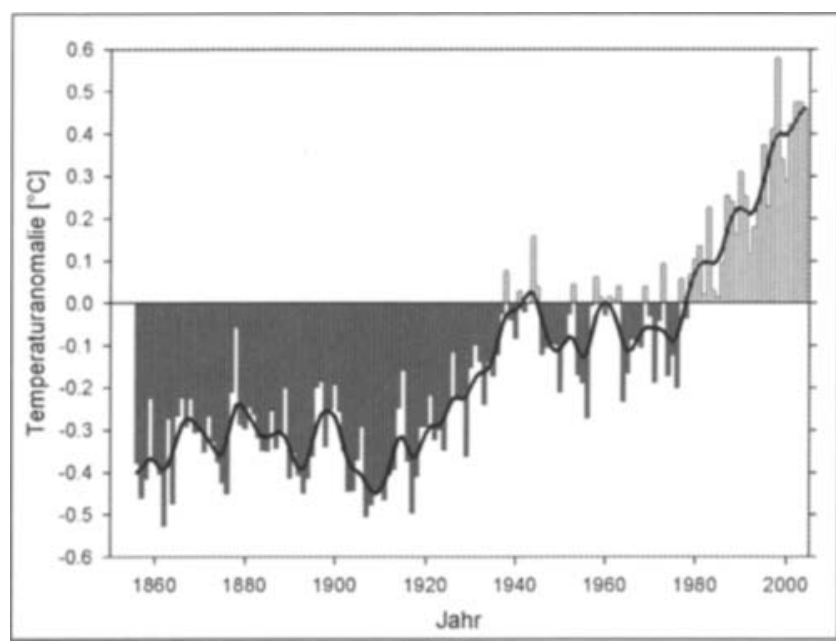

wurde Ziel des „Zwischenstaatlichen Ausschusses zum Klimawandel" (Intergovernmental Panel on Climate Change, IPCC), der 1988 unter Federführung zweier UN-Organisationen, UNEP (United Nations Environment Program) und WMO (World Meteorological Organization) gegründet wurde. Der IPCC erstellt regelmäßige Zusammenfassungen der aktuellen Forschungsergebnisse, um bei der Formulierung von Zielen für die globale Umweltpolitik, wie z.B. dem Kyoto-Protokoll, zu unterstützen. Dazu gehören auch Projektionen von möglichen zukünftigen Klimaentwicklungen mit Hilfe detaillierter Klimamodelle, gestützt auf vorgegebene Szenarien zum Ausstoß von C02 und anderen Treibhausgasen.

Die bis heute neueste Serie von IPCC- Szenarien (Hauptszenarien A1, B1, A2, B2) folgt abgestimmten möglichen Entwicklungslinien, so genannten Storylines, die unterschiedlichen Entwicklungen der Weltwirtschaft, des Bevölkerungswachstums und anderer Faktoren folgen (Nakicenovic et al. 2000). Die zugehörigen Emissionsentwicklungen für $\mathrm{CO}_{2}$ und $\mathrm{SO}_{2}$ von 2000 bis 2100 unterscheiden sich in ihrer Stärke ebenso wie in ihrem zeitlichen Verlauf. Diese Emissionen werden in die globalen und regionalen Klimamodelle eingespeist und bewirken durch zahlreiche nicht-lineare Wechselwirkungen Veränderungen des globalen und regionalen Klimas.

Die Szenarienfamilie A2 beschreibt eine sehr heterogene Welt. Die Grundannahmen sind Autarkie und die Bewahrung von lokalen Identitäten. Wirtschaftliches Wachstum ist vor allem regional orientiert. Die Szenarienfamilie A1 beschreibt eine künftige Welt mit sehr raschem wirtschaftlichem Wachstum, einer Weltbevölkerung, die Mitte des 21. Jahrhunderts zahlenmäßig ihren Höhepunkt erreicht und danach abnimmt, und der raschen Einführung von neuen und effizienteren Technologien. Die drei A1-Gruppen unterscheiden sich durch ihren jeweiligen technologischen Schwerpunkt: intensive Nutzung fossiler Brennstoffe
(A1FI), nicht-fossiler Energiequellen (A1T) oder Ausgeglichenheit über alle Energieträger hinweg (A1B). Die Szenarienfamilie $B 1$ beschreibt eine konvergierende Welt mit der gleichen globalen Bevölkerung wie im Al Szenario, aber mit raschen Veränderungen in den wirtschaftlichen Strukturen hin zu einer Dienstleistungs- und Informationswirtschaft, mit deutlich geringerer Materialintensität und Einführung von emissionsarmen und ressourcenschonenden Technologien. Das Schwergewicht liegt auf globalen Lösungen in Richtung wirtschaftlicher, sozialer und ökologischer Nachhaltigkeit, einschließlich verbesserter Gerechtigkeit, aber ohne zusätzliche Klimaschutzinitiativen.

\section{Mögliche Klimaänderungen in Europa}

Eine detaillierte Darstellung globaler Klimaänderungen, globaler Klimamodelle und ihrer Unsicherheiten befindet sich im neuesten IPCC Bericht (Solomon 2007). Die deutschen IPCC Klimarechnungen wurden mit Klimamodellen des Max-Planck-Instituts für Meteorologie durchgeführt. Das MPI-M IPCC-Klimamodell besteht aus zwei Hauptkomponenten, dem ECHAM5 Atmosphärenmodell, das auch die Landkomponente beinhaltet, und dem MPIOM Ozeanmodell. Diese Komponenten werden durch einen Koppler miteinander verbunden. Die horizontale Auflösung des Atmosphärenmodells beträgt ca. $1,9^{\circ}$, jene des Ozeanmodells $1,5^{\circ}$ in geographischer Länge und Breite. Das IPCC-Klimamodell beinhaltet die aufgelösten und parametrisierten Prozesse, die für die Budgets von Impuls, Energie und Wasser im Erdsystem von Bedeutung sind. Wichtige Treibhausgase wie z.B. $\mathrm{CO}_{2}$ werden extern vorgeschrieben.

Die Simulation des Klimas im 20. Jahrhundert und der zukünftigen szenarienabhängigen Klimaverläufe erfordert sorgfältige Tests der eingesetzten Klimamodelle. Diese Modelle sind entwickelt worden, um das Klima der letzten Jahrzehnte möglichst genau simulieren zu können. Nur für diese wenigen Jahrzehnte sind genügend umfangreiche direkte Beobachtungen von Atmosphäre und Ozean verfügbar, die eine genaue Prüfung von Klimamodellen erlauben. Der Rechtfertigung für den Einsatz dieser Modelle in geänderten Klimabedingungen beruht also allein auf dem Erfolg dieser Modelle in der Simulation des Klimas der letzten Jahrzehnte. Die durch geänderte Klimabedingungen, z.B. erhöhtem $\mathrm{CO}_{2}$ - Gehalt in der Atmosphäre, im Klimamodell simulierte Klimaänderung, z.B. in der global gemittelten Temperatur, ist eine Eigenschaft, die sich von Klimamodell zu Klimamodell unterscheiden kann, selbst bei gleicher Simulationsgüte des bekannten vergangenen Klimas.

Die Tests von Klimamodellen verlaufen in der Regel in zwei Stufen. Zuerst werden die einzelnen Modellkomponenten entwickelt und getestet, bis die erforderliche Güte erreicht worden ist. Atmosphärenmodelle werden beispielsweise mit vorgegebenen, aus Beobachtungen abgeleiteten Randbedingungen der 80 er und $90 \mathrm{er}$ Jahre angetrieben und das 
simulierte Klima wird mit dem beobachteten Klima dieser Jahre verglichen. In der zweiten Stufe werden die gekoppelten Klimamodelle in Simulationen über mehrere Jahrzehnte oder wenige Jahrhunderte getestet. Es wird dabei vor allem auf die Stabilität des Klimas und auf jene Phänomene geachtet, die direkt von den Kopplungsprozessen abhängen, wie z.B. die jahreszeitlich abhängige Eisverteilung in der Arktis und Antarktis, oder EI Niño/La Niña im tropischen Pazifik. Mit dem MPI-M IPCC-Modell wurde eine Simulation über mehr als 500 Jahre mit konstanten vorindustriellen Klimabedingungen durchgeführt, in der sich ein stabiler Klimaverlauf gezeigt hat. Die global gemittelte Bodentemperatur ändert sich dabei um weniger als $0,03^{\circ} \mathrm{C}$ pro Jahrhundert. Diese lange Simulation des vorindustriellen Klimas wurde als Basis für die Simulationen des Klimas des 20. Jahrhunderts und die anschließenden szenarienabhängigen Klimarechnungen verwendet.

Um die Auswirkungen globaler Klimaänderungen auf Regionen in Europa zu untersuchen, wird ein regionales Klimamodell in das globale Klimamodell eingebettet. So ist es möglich, wie mit einer Lupe eine spezielle Region viel detaillierter zu untersuchen und eine Brücke zwischen globalen Klimaänderungen und lokalen Konsequenzen zu schlagen.

Für diese Studien wurde das am Max-Planck-Institut für Meteorologie (MPI-M) entwickelte regionale Modell REMO in das globale Klimamodell ECHAM5/MPI-OM des MPI-M integriert, d.h. an den seitlichen Rändern des Untersuchungsgebietes strömen Luftmassen, wie sie im Globalmodell berechnet wurden, in das Berechnungsgebiet des regionalen Modells hinein. In REMO wird dann ein regionales Klima unter Berücksichtigung der globalen Infor- mationen und der lokalen und regionalen Gegebenheiten ausgerechnet. Das hierfür eingesetzte regionale Klimamodell REMO (Jacob 2001, Jacob et al. 2007) zeigt die Klimaentwicklung des vergangenen Jahrhunderts recht realitätsnah, wie der Vergleich zu Beobachtungen - auch in stark strukturiertem Gelände wie den Alpen (Frei et al. 2003) ergibt. Diese Überprüfung ist notwendig, um die Güte der Modellergebnisse zu bewerten. Die regionalen Modellergebnisse sind weitgehend ähnlich zu den mit dem globalen Modell ausgerechneten. Durch die höhere horizontale Gitterauflösung liefert das regionale Modell jedoch wesentlich mehr kleinräumige Informationen, die regional auch durch die Beschaffenheiten des Untergrundes (Hügeligkeit, Bewuchs) abweichen können. Zusätzlich wird eine wesentlich höhere Genauigkeit bei der Simulation seltener kleinräumiger Extremereignisse erreicht (z.B. sommerliche Starkniederschläge).

Für eine mögliche Projektion des Klimas bis 2100 wird REMO zunächst mit einer Gitterweite von $50 \mathrm{~km}$ für Europa betrieben. So können mögliche unterschiedliche Entwicklungen in Nord-, Zentral- und Südeuropa erfasst werden. Alle Klimasimulationen - global und regional wurden mit Unterstützung des Deutschen Klimarechenzentrum (DKRZ) durchgeführt.

Exemplarisch ist für das A1B Szenario aus Abbildung $2 \mathrm{zu}$ erkennen, dass die Temperaturerhöhung in Europa regional unterschiedlich stark ausfallen wird. Schon für die Dekade 2040 bis 2050 wird für den Mittelmeerraum ein Anstieg der Sommertemperaturen (Abb.2, unten links) von mehr als 2,5 im Vergleich zu 1961 bis 1990 berechnet, während in weiten Teilen Zentraleuropas nur weniger als $1^{\circ}$ Erwärmung erwartet wird. In den Wintermonaten (Abb.

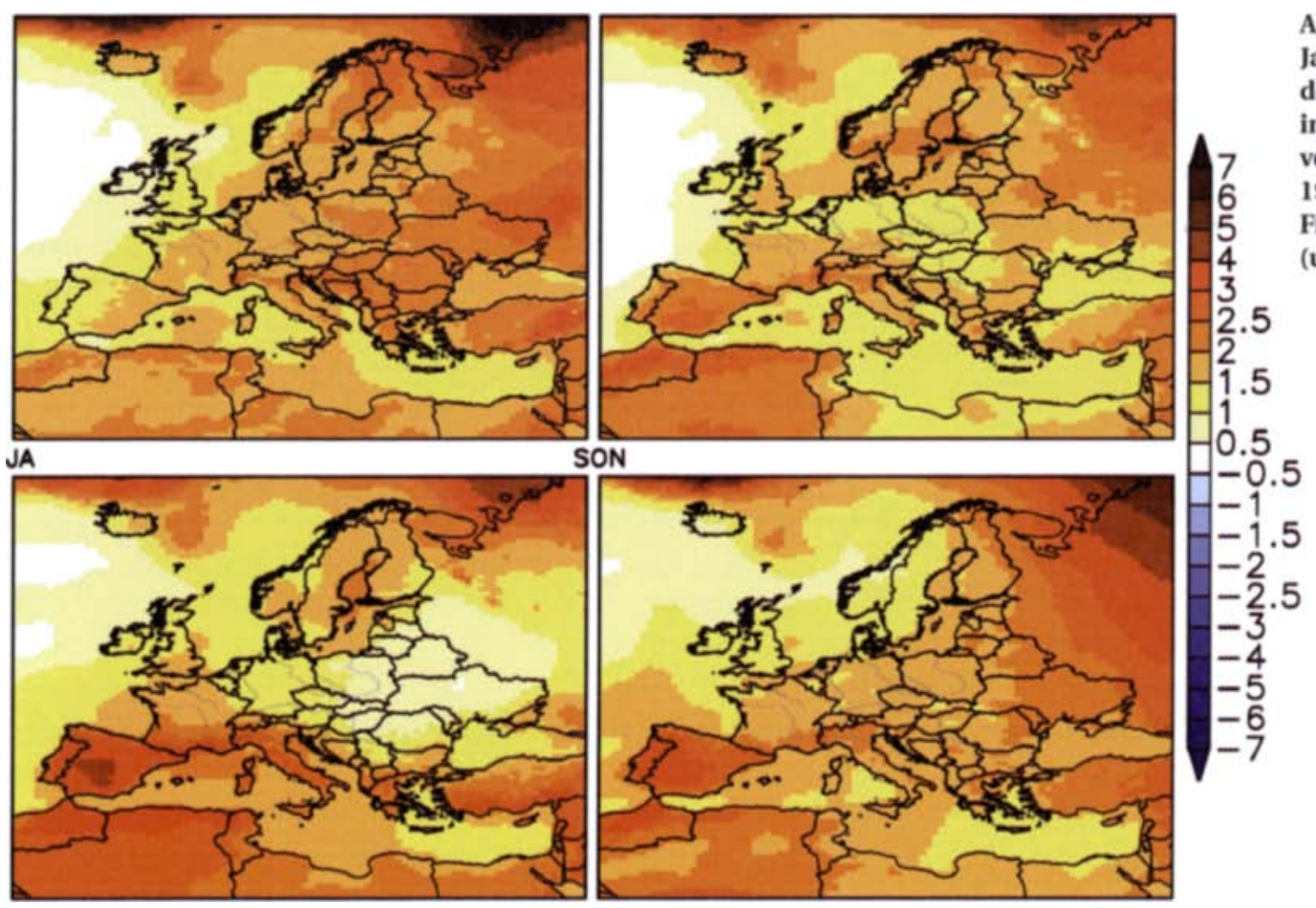

Abbildung 2

Jahreszeitliche Abweichung der Mitteltemperatur $\left({ }^{\circ} \mathrm{C}\right)$ in $\mathbf{2} \mathbf{m}$ über Grund um 2050 von der Referenzperiode 1961 bis 1990. Winter (o.1.), Frühjahr (o.r.), Sommer (u.l.) und Herbst (u.r.) 

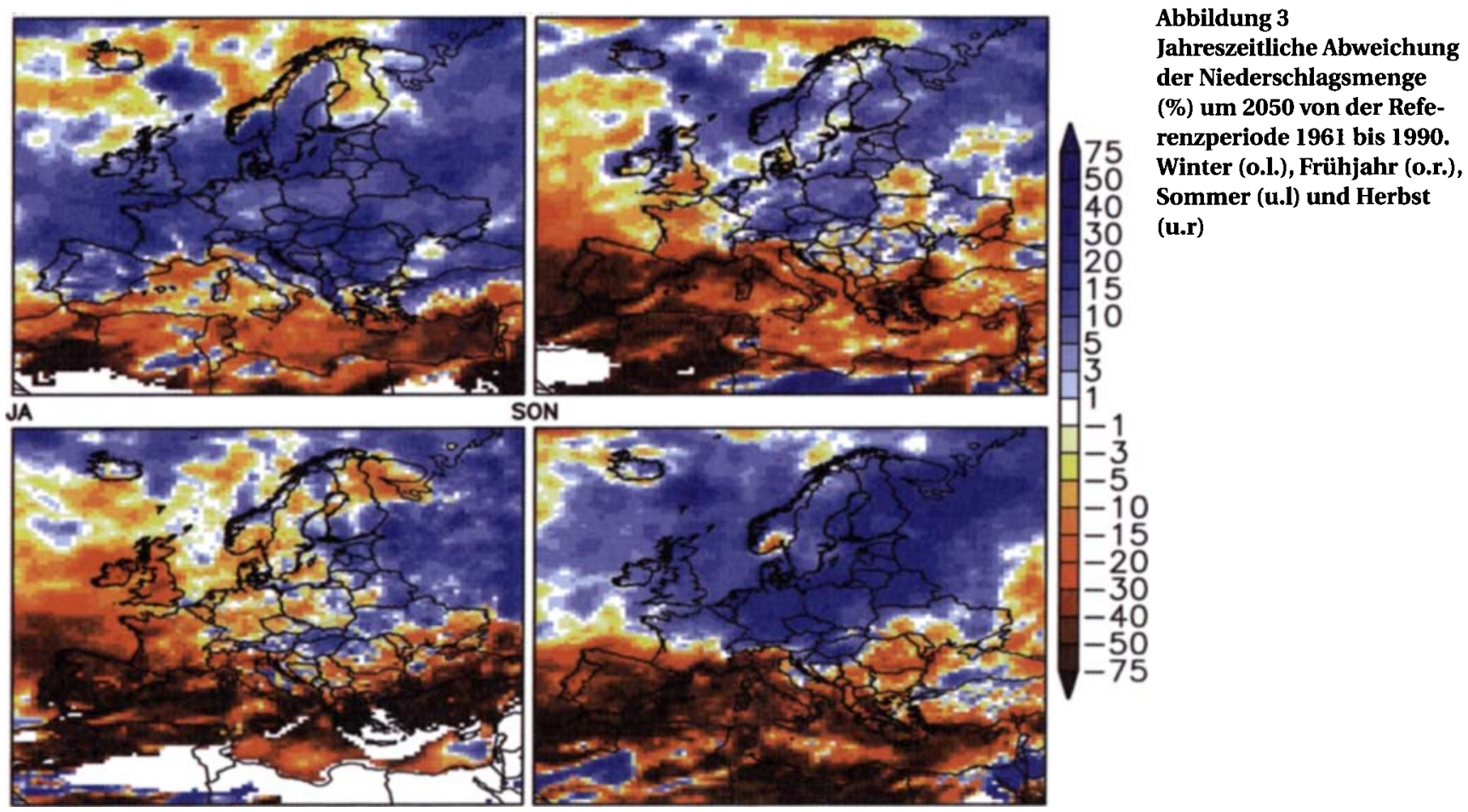
Abbildung 3 Sommer (u.l) und Herbst

2, oben links) hingegen beträgt die berechnete Temperaturerwärmung ca. $1,5^{\circ}$ bis $2^{\circ}$ und erstreckt sich von Skandinavien bis zum Mittelmeer. Nur in Regionen unter direktem Einfluss des Atlantiks (Großbritannien, Portugal, Teile von Spanien) fällt im Winter der Temperaturanstieg geringer aus. Die Verhältnisse in der letzten Dekade des Jahrhunderts (hier nicht dargestellt) zeigen eine großräumige Erwärmung des gesamten Kontinents um mehr als $3^{\circ}$ im Sommer und Winter.

Gleichzeitig mit den projizierten Temperaturänderungen werden Niederschlagsänderungen berechnet. Schon zur Mitte des 21. Jahrhunderts zeigt sich ein deutlicher Trend zur Niederschlagsabnahme von bis zu 50\% und mehr im Mittelmeerraum in allen Jahreszeiten, während insbesondere Skandinavien im Winter mit mehr Niederschlag rechnen muss (Abb.3). Für die Sommermonate wird nach dem AlB Szenario auch in weiten Teilen Nord- und Zentraleuropas ein Niederschlagsrückgang (z. B. Großbritannien mehr als $30 \%$ ) ermittelt. Dies scheint mit einer Verlagerung des Azorenhochs nach Nordosten verbunden zu sein, das die großräumigen Strömungen im Vergleich zu heute verschiebt. Hierzu sind weitere Untersuchungen geplant. Die schon bis 2050 berechneten Trends verstärken sich zum Ende des Jahrhunderts in ganz Europa mit einer weiträumigen Niederschlagsabnahme im Sommer bis nach Südskandinavien (nicht gezeigt).

Beispielhaft für extreme Situationen wurde die Entwicklung der Anzahl der Frosttage $\left(\operatorname{Tmin}<0^{\circ} \mathrm{C}\right.$ ) und der heißen Tage ( $\operatorname{Tmax}>30^{\circ} \mathrm{C}$ ) untersucht: In ganz Europa kann schon bis 2050 die Zahl der Frosttage deutlich abnehmen. Gleichzeitig nimmt die Zahl der heißen Tage im A1B-Szenario bis 2100 um 5 bis 50 Tage zu. Nur für England und
Skandinavien wird keine Zunahme der heißen Tage berechnet.

\section{Deutschland ist von möglichen Klimaänderungen unter- schiedlich stark betroffen:}

Im Auftrag des Umweltbundesamtes (UBA) hat das MPI-M mit Unterstützung des Deutschen Klimarechenzentrums Szenarien für mögliche Klimaänderungen in Deutschland bis zum Jahr 2100 erarbeitet (Jacob et al. 2008). Die Klimasimulationen mit REMO wurden mit einer räumlichen Auflösung von $10 \mathrm{~km}$ durchgeführt. Hierbei liefern diese Simulationen Erkenntnisse, die es bislang noch nicht in dieser Detailliertheit gab.

Die Ergebnisse dieser Klimasimulationen lassen sich auf folgende Aussagen verdichten: Je nach Veränderungen der Treibhausgase könnten bis zum Ende des Jahrhunderts die Temperaturen in Deutschland - vor allem im Süden und Südosten - um mehr als $4^{\circ} \mathrm{C}$ im Vergleich zu den letzten 50 Jahren steigen. Im Sommer kann es in weiten Teilen Deutschlands weniger Niederschläge geben. Im Winter könnten im Süden und Südosten mehr Niederschläge fallen, wobei allerdings wegen der gestiegenen Temperaturen weniger Schnee fallen kann. Das langjährige Mittel der Jahresniederschlagsmenge scheint jedoch etwa gleich zu bleiben.

Die Ergebnisse im Detail: Mehr Treibhausgase können in Deutschland zu einer mittleren Erwärmung führen, die im Jahr 2100 - abhängig von der Höhe zukünftiger Treibhausgasemissionen - zwischen $2,5^{\circ} \mathrm{C}$ und $3,5^{\circ} \mathrm{C}$ liegt (Abb. 4 , oben). Die Entwicklung der jährlichen Niederschlagssum- 


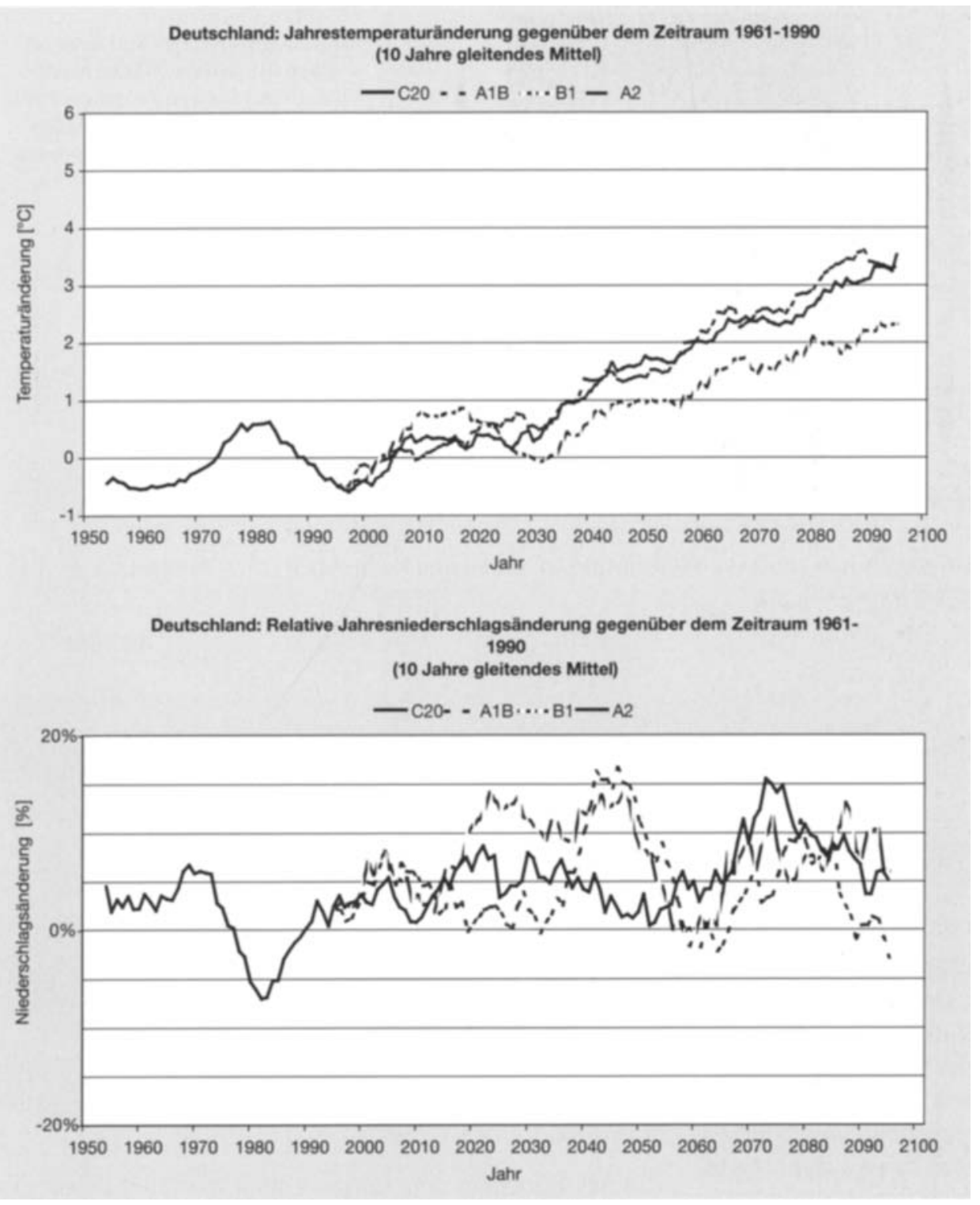

Abbildung 4

Mittlere Jahrestemperaturänderung (oben) und mittlere relative Niederschlagsänderung (unten) zur Klimanormalperiode 19611990 in Deutschland als gleitendes 10-Jahresmittel men zeigt eine ausgeprägte dekadische Variabilität, jedoch keinen generellen Trend (Abb. 4, unten). Diese Erwärmung wird sich saisonal und regional unterschiedlich stark ausprägen. Am stärksten dürften sich der Süden und Südosten Deutschlands im Winter erwärmen. Bis zum Jahr 2100 könnten die Winter hier um mehr als $4^{\circ} \mathrm{C}$ wärmer werden als im Zeitraum 1961 bis 1990.

Gleichzeitig könnten in Zukunft - im Vergleich zum Zeitraum 1961 bis 1990 - die sommerlichen Niederschläge großflächig abnehmen. Besonders stark gehen in den Simulationen die Sommerniederschläge in Süd- und Südwest-Deutschland sowie in Nordost-Deutschland zurück (Abb. 5). Hier könnte es bis zum Ende dieses Jahrhunderts im Vergleich zu heute ein Minus von bis zu 30 Prozent bei den Sommerniederschlägen geben. Im Gegensatz hierzu könnte im Winter ganz Deutschland feuchter werden (Abb.5). Vor allem in den Mittelgebirgen Süd- und Südwest-Deutschlands ist über ein Drittel mehr Niederschlag zu erwarten als heute. Blickt man zum deutschen Küsten- raum, so fällt auf, dass bis zum Jahr 2100 die Erwärmung der Ostseeküste mit $2,8^{\circ} \mathrm{C}$ etwas stärker sein könnte als die der Nordseeküste $\left(2,5^{\circ} \mathrm{C}\right)$. Obwohl sich an beiden Küsten die jährliche Niederschlagsmenge nicht ändert, dürfte den Touristen gefallen, dass es im Sommer bis zu $25 \%$ weniger regnen könnte. Im Winter gibt es jedoch bis zu 30\% mehr Niederschlag.

Weitere Analysen und Darstellungen möglicher Klimaänderungen in Deutschland befinden sich in Jacob et al. 2008. Dort werden Ergebnisse weiterer Emissionsszenarien dargestellt, zusätzliche meteorologische Größen untersucht und Indizes aufgelistet. Tab. 1 enthält einige Beispiele und wurde aus Jacob et al. 2008 entnommen.

REMO simuliert gut extreme Temperaturereignisse. Die Szenarien zeigen eine deutliche Zunahme sowohl der Intensität als auch der Häufigkeit von Temperatur-Extremereignissen, wobei prozentual die seltenen Ereignisse den stärksten Zuwachs aufweisen. Die Simulation von Nieder- 

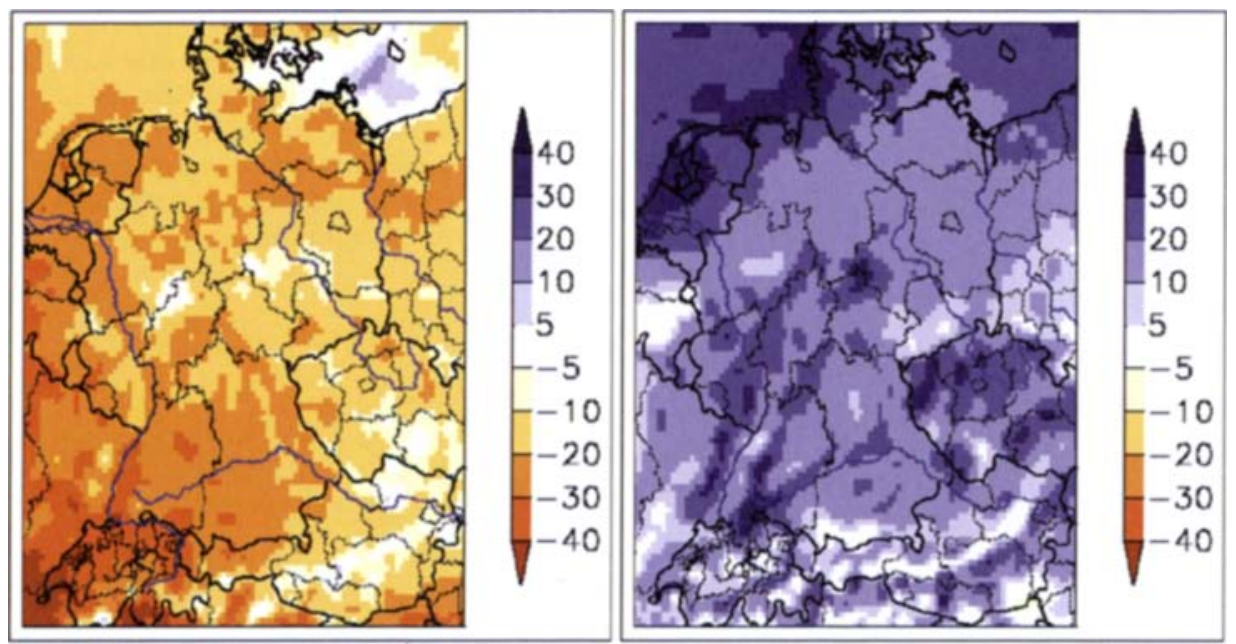

Abbildung 5

Änderung (\%) der Sommerniederschläge (li) und der Winterniederschläge (re) für den Zeitraum 2071 bis 2100 im Vergleich zu 1961 bis 1990 in Deutschland in gleitenden 10 Jahresmitteln.

Tabelle 1

Mittlere jährliche Kennzahlen des Kontrolllaufs (C20) für 1961-1990 und die Änderung zum Kontrolllauf (2071-2100 minus 1961-1990) für alle 3 Szenarien (Modellgebietsmittel)

\begin{tabular}{|l|c|c|c|c|}
\hline & C20 & A1B - C20 & B1 - C20 & A2- C20 \\
\hline Absolutes Temperaturmaximum & 36,3 & 4,0 & 3,2 & 6,5 \\
\hline Hunmindex (Sommer; JJA) & 26,6 & 2,3 & 1,6 & 2,3 \\
\hline Frosttage/Jahr & 45,3 & $-33,1$ & $-24,9$ & $-32,0$ \\
\hline Gradtagszahl & 3788,0 & $-1068,3$ & $-727,37$ & $-1026,6$ \\
\hline GröBte 5-Tages-Niederschlagssumme / Jahr & 73,8 & 5,8 & 4,5 & 5,8 \\
\hline GröBte 1-Tages-Niederschlagssumme / Jahr & 40,1 & 7,7 & 5,6 & 7,0 \\
\hline
\end{tabular}

schlagsextremen wurde bis jetzt noch nicht systematisch mit Messdaten verglichen. Bei den Starkniederschlägen ist, über das ganze Jahr gesehen, keine deutliche Zunahme der Häufigkeit von Extremereignissen zu erwarten. Die Intensität der Starkniederschläge nimmt jedoch zu.

Wegen gleichzeitig steigender Wintertemperaturen in den Alpen - bis zum Ende des Jahrhunderts könnten es mehr als $4^{\circ} \mathrm{C}$ sein - wird der Niederschlag häufiger als Regen denn als Schnee fallen. Fiel in der zweiten Hälfte des 20. Jahrhunderts dort im Jahr etwa ein Drittel des Gesamtniederschlags als Schnee, könnte es bis Ende des 21. Jahrhunderts nur noch ein Sechstel sein. Diese Veränderungen haben zur Folge, dass sich die Anzahl der Schneetage pro Jahr reduziert, und zwar stärker in niedrigen Regionen wie z. B. Garmisch-Partenkirchen und Mittenwald, für die Abnahmen um deutlich mehr als die Hälfte möglich sein können. In den höheren Regionen wie Zermatt und St. Moritz wurde jedoch nur eine Reduktion um ca. ein Drittel berechnet.

Bis zum Ende des 21. Jahrhunderts könnten daher die schneebedeckten Flächen im Alpenraum sehr stark schrumpfen, wenn die Erwärmung stark zunimmt (z.B. $>4^{\circ} \mathrm{C}$. Doch auch schon bei einer Temperaturzunahme von $3^{\circ} \mathrm{C}$, wie sie bis zur Mitte des 21. Jahrhunderts si- muliert wird, können sehr große schneebedeckte Flächen verschwinden, die heute noch als schneesicher gelten (Abb. 6).

Diese schnellen und tiefgreifenden Veränderungen des Klimas in Deutschland können gravierende Folgen für die Menschen und die Umwelt haben. Die Schadenspotentiale extremer Wetterereignisse wie Hitzewellen, Starkniederschläge und Stürme sind oftmals noch wesentlich größer als jene der schleichenden Klimaänderungen. Deswegen sind zurzeit am MPI-M detaillierte Analysen der Klimaszenarien in Arbeit, um Aussagen zur Häufigkeit und Stärke künftiger Extremereignisse machen zu können.

Abbildung 7 zeigt hierzu eine zu erwartende Veränderung der Anzahl an Sommertagperioden (zusammenhängende Tage mit einer Maximaltemperatur $>25^{\circ} \mathrm{C}$ ) für das SRES B2 Emissionsszenario für drei europäische Einzugsgebiete (Nordeuropa: Luleaelven, Mitteleuropa: Rhein, Südeuropa: Ebro). Hiernach ist in allen Gebieten für den Zeitraum 2071-2100 gegenüber dem Referenzzeitraum 1961-1990 mit einer deutlichen Häufigkeitszunahme insbesondere der langen Perioden zu rechnen.

Im Einzugsgebiet des Luleaelven steigt die Häufigkeit von Perioden der Längen 3-10 Tage (11-20 Tage) von 2 auf 10 


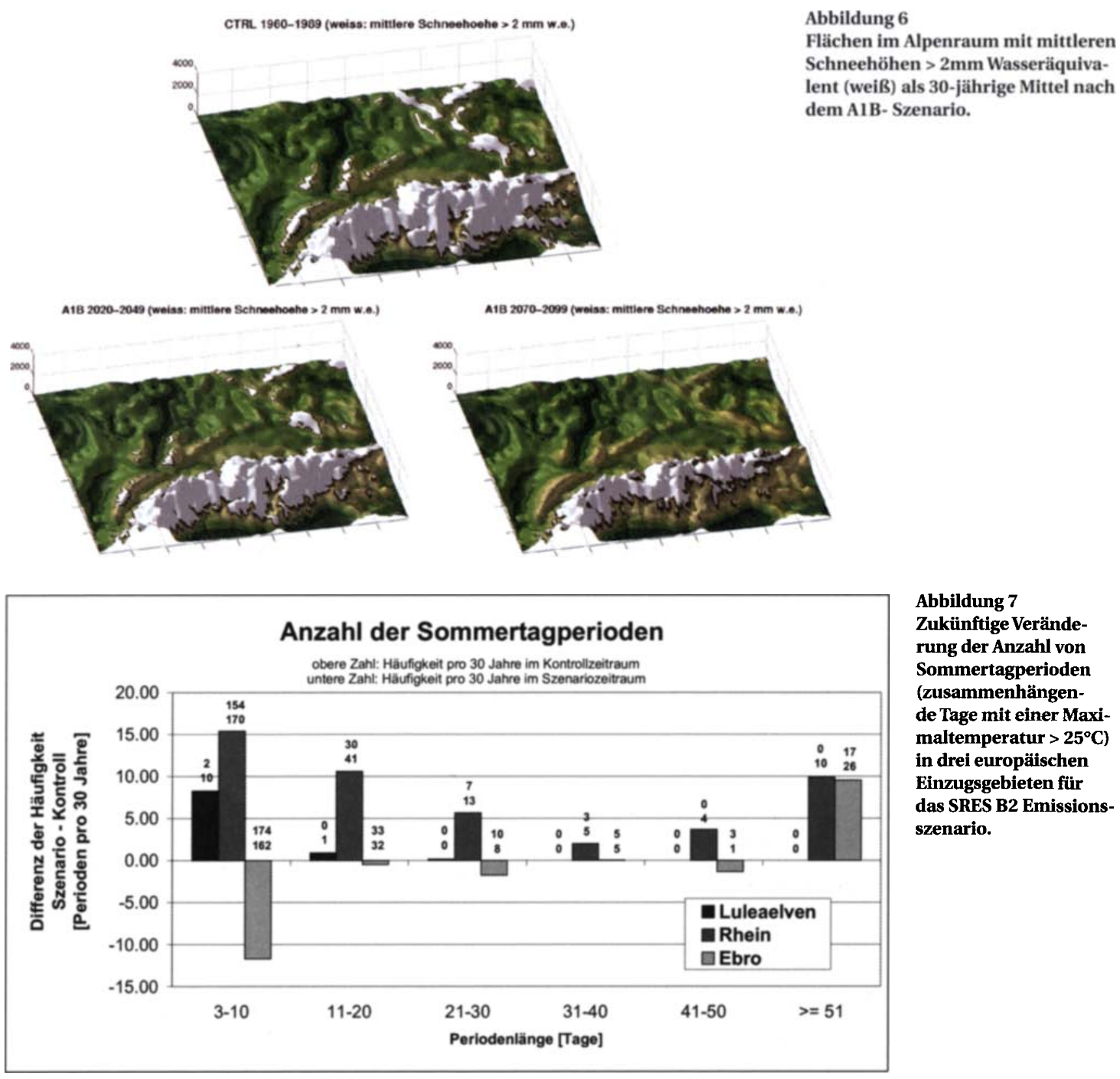

(von 0 auf 1) Ereignisse pro 30 Jahre. Sommertagperioden mit einer Länge von mehr als 20 Tagen treten im "kalten“ Skandinavien auch im Klimaszenario nicht auf.

Anders verhält es sich im Rhein- und Ebroeinzugsgebiet. Am Rhein zeigen sich im Klimaszenario Periodenlängen $>40$ Tage. Derartige lange und heiße Zeiträume treten im berechneten Kontrollklima, das die heutigen klimatischen Bedingungen repräsentiert, nicht auf. Auch im Ebroeinzugsgebiet steigt die Anzahl langer, heißer Perioden mit Längen > 51 Tage deutlich von 17 auf 26 Ereignisse pro 30 Jahre. Hier muss in der Zukunft also fast jedes Jahr mit langen Hitzeperioden gerechnet werden.

Die beschriebenen Veränderungen in Anzahl und Länge von Hitzeperioden werden unweigerlich mit einer erhöhten Auftrittswahrscheinlichkeit von Niedrigwasserereignissen verbunden sein. Für den Pegel Kaub (Rhein) erga- ben erste Analysen aus der klimatologischen Wasserbilanz für das B2 Emissionsszenario hierzu eine deutliche Häufigkeitszunahme von möglichen Niedrigwasserperioden (definiert als zusammenhängende Tage mit einem mittleren Abfluss $<750 \mathrm{~m}^{3} / \mathrm{s}$ ) bis zu 21 Tagen Länge.

\section{Schlusswort}

Alle oben erwähnten Ergebnisse entstammen je einer Simulation pro Emmissionsszenario. Um die natürliche Variabiltät berücksichtigen zu können, müssten viele dieser möglichen Realisationen eines Emmissionsszenarios berechnet werden. Dies ist auch geplant und wird dann verwendet, um die Robustheit der Klimaänderungsmuster zu analysieren. 


\section{Literatur}

Solomon, S.; Qin, D.; Manning, M.; Chen, Z.; Marquis, M.; Averyt, K.B.; Tignor, M. and Miller, H.L. (eds.) (2007): Climate Change: The Physical Science Basis. Contribution of Working Group I to the Fourth Assessment Report of the Intergovernmental Panel on Climate Change. Cambridge University Press, Cambridge and New York, 996 pp. www.ipcc.ch

Frei, C.; Christensen, J.H.; Deque, M.; Jacob, D.; Jones, R.G.; Vidale, P.L. (2003): Daily precipitation statistics in regional climate models: Evaluation and intercomparison for the European Alps. J. Geophys. Res. 108 (D3), 4124, doi:10.1029/2002JD002287.

Jacob, D. (2001): A note to the simulation of the annual and interannual variability of the water budget over the Baltic Sea drainage basin'. Meteorol Atmos Phys 77, S. 61-73.

Jacob, D.; Bärring, L.; Christensen, O.B.; Christensen, J.H.; Hagemann, S.; Hirschi, M.; Kjellström, E.; Lenderink, G.; Rockel, B. Schär, C.; Seneviratne, S.I.; Somot, S.; van Ulden, A. and van den Hurk, B. (2007): An inter-comparison of regional climate models for Europe: Design of the experiments and model performance. Prudence Special Issue, Climatic Change, Vol.81, Supplement 1, May 2007.
Jacob, D.; Göttel, H.; Kotlarski, S.; Lorenz, Ph; Sieck, K. (2008): Klimaauswirkungen und Anpassung in Deutschland - Phase 1: Erstellung regionaler Klimaszenarien für Deutschland. Abschlussbericht zum UFOPLAN-Vorhaben 2044113.

Nakicenovic, N.; Alcamo, J.; Davis, G.; de Vries, B.; Fenhann, J.; Gaffin, S.; Gregory, K.; Grübler, A.; Jung, T.Y.; Kram, T.; La Rovere, E.L.; Michaelis, L.; Mori, S.; Morita, T.; Pepper, W.; Pitcher, H.; Price, L.; Raihi, K.; Roehrl, A.; Rogner, H-H.; Sankovski, A.; Schlesinger, M.; Shukla, P.; Smith, S.; Swart, R.; van Rooijen, S.; Victor, N. and Dadi, Z. (2000): IPCC Special Report on Emissions Scenarios. Cambridge University Press, Cambridge and New York.

Alle Abbildungen: Max-Planck-Institute for Meteorology

Wir danken Katharina Bülow, Holger Göttel, Stefan Hagemann und Philip Lorenz vom Max-Planck-Institut für Meteorologie in Hamburg, die zur Durchführung der Modellrechungen, der Erstellung der Abbildungen und Auswertungen der REMO- Ergebnisse beigetragen haben.

Dr. Daniela Jacob

Max-Planck-Institute for Meteorology

Bundesstrasse 53

20146 Hamburg

Telefon: +49(0) $40-41173313$

Fax: $\quad+49(0) 40-41173357$

Mail: daniela.jacob@zmaw.de 